\title{
Coagulopathy of peritoneovenous shunts: studies on the pathogenic role of ascitic fluid collagen and value of antiplatelet therapy
}

\author{
H H SALEM, F J DUDLEY, A MERRETT, \\ JEAN PERKIN, AND B G FIRKIN \\ From the Department of Medicine, Monash University Medical School, and the Gastroenterology Service, \\ Alfred Hospital, Victoria, Australia
}

SUMMARY The role of ascitic fluid collagen in the pathogenesis of the coagulopathy that follows peritoneovenous shunting was examined. Collagen was partially purified from ascitic fluid and infused into rabbits. All animals developed changes in their haemostatic profile consistent with intravascular coagulation. Aspirin therapy, for five days before the collagen infusion, prevented these changes. Seven patients undergoing a total of eight peritoneovenous shunts for intractable ascites received antiplatelet therapy (aspirin and dipyridamole) in the immediate pre- and postoperative period. After six shunts no thrombocytopenia or prolongation of clotting times developed to suggest decompensated consumptive coagulopathy. Complicating factors may have contributed to the deterioration in haemostasis in the other two patients. There was no early shunt occlusion. The results support the hypothesis that ascitic fluid collagen is important in the pathogenesis of intravascular coagulation postascitic fluid infusion and indicate that antiplatelet drugs may be of value in preventing this complication.

Disseminated intravascular coagulation invariably follows the insertion of peritoneovenous (Le Veen) shunts. ${ }^{1-3}$ The coagulopathy is clinically significant in $20-50 \%$ of patients and often necessitates ligation of the shunt. ${ }^{24}$ Although procoagulants, particularly thromboplastin, were considered major mediators of this complication, ${ }^{56}$ the failure of other studies to detect such substances in ascitic fluid $^{7} 8$ suggested that alternative factors were involved. It has been recently shown that ascitic fluid contains a platelet aggregating factor identified as being collagen. ${ }^{8}$ It was suggested that this material, by activating both platelets and clotting factors, could be aetiologically significant in the pathogenesis of disseminated intravascular coagulation complicating peritoneovenous shunts.

The aim of this study was to confirm that the infusion of collagen purified from ascitic fluid caused disseminated intravascular coagulation in experimental animals, and examine the effects of aspirin

Address for correspondence: Dr F J Dudley, Gastroenterology Service, Alfred Hospital, Commercial Road. Prahran, 3181. Victoria, Australia.

Received for publication 9 August 1982 pretreatment on this complication and study the efficacy of antiplatelet therapy in controlling disseminated intravascular coagulation in patients receiving peritoneovenous shunts for refractory ascites.

\section{Methods}

PURIFICATION OF COLLAGEN FROM ASCITIC FLUID Collagen was partially purified by neutral salt precipitation $^{9}$ from four litres of ascitic fluid obtained from a patient with chronic liver disease at the time of insertion of a peritoneovenous shunt. In all the steps described below centrifugation was undertaken at $20000 \mathrm{~g}$ for 30 minutes and all purification procedures including centrifugation were performed at $4^{\circ} \mathrm{C}$. The cellular component of the fluid was separated by centrifugation and the cell free supernatant dialysed for $\mathbf{4 8}$ hours against four changes of $0.02 \mathrm{M} \mathrm{Na}{ }_{2} \mathrm{HPO}_{4}, \mathrm{pH} \mathrm{7.4}$. The collagen rich precipitate was collected by centrifugation, resuspended in $0 \cdot 1 \mathrm{M}$ acetic acid and dialysed against the same acid concentration for a further $\mathbf{4 8}$ hours. Under these conditions collagen became soluble and 
any residual precipitate was removed by centrifugation. Collagen was reprecipitated from the supernatant using $\mathrm{NaCl}$ at a final concentration of $5 \%$ $(\mathrm{w} / \mathrm{v})$, and complete precipitation allowed to proceed for four hours. The precipitate was collected by centrifugation, resuspended in normal saline and stored at $-20^{\circ} \mathrm{C}$ in $2 \mathrm{ml}$ aliquots containing a total of $50 \mathrm{mg}$ of protein assayed by the Lowry technique. ${ }^{10}$ The presence of collagen in the final preparation was confirmed by sodium dodecyl sulphate polyacrylamide gel electrophoresis. ${ }^{8}$

\section{COAGULATION STUDIES}

The prothrombin, thrombin, Stypven, and activated partial thromboplastin times were performed by standard methods. ${ }^{11}$ Factor VIII and IX clotting activities were assayed by a one stage technique ${ }^{11}$ using commercial deficient substrates (Dade, Miami, Florida). Antithrombin III levels were measured by a two stage assay using the thrombin specific chromogenic substrate S2238. ${ }^{12}$ Fibrinogen was assayed by the fibrin clot weight method. ${ }^{11}$ Fibrin monomers were detected by the protamine sulphate method ${ }^{11}$ and fibrin degradation products by the latex agglutination technique. ${ }^{11}$ Platelets were counted manually using phase contrast microscopy. The partially purified ascitic fluid collagen preparation was diluted 1:20 with normal saline $(0.9 \%)$ for platelet aggregation studies. ${ }^{8}$

\section{ANIMAL EXPERIMENTS}

A total of 14 male New Zealand rabbits were studied. The rabbits were 3-6 months old and weighed an average of $3 \mathrm{~kg}$.

Twelve rabbits were infused with $2 \mathrm{ml}$ of the partially purified ascitic fluid collagen suspension containing $50 \mathrm{mg}$ protein. In six, soluble aspirin (15 $\mathrm{mg} / \mathrm{kg}$ body weight) dissolved in water was administered orally, by pipette, for five days before study. Two rabbits served as controls and received $2 \mathrm{ml}$ normal saline $(0.9 \%)$ intravenously, instead of the collagen preparation. The animals were anaesthetised with pentobarbitone $(40 \mathrm{mg} / \mathrm{kg}$ body weight), after which the carotid artery was exposed and cannulated with a 21 gauge silastic cannula. A pump controlled infusion of acid citrate dextrose was started at a set rate of $1.5 \mathrm{ml} / \mathrm{h}$ through the cannula. Blood was collected for baseline coagulation studies from the carotid cannula, and $2 \mathrm{ml}$ of collagen suspension injected over 15 minutes through a marginal ear vein. At half hourly intervals, and for two hours, blood samples were collected from the carotid cannula. On each occasion, the cannula was disconnected from the pump, the first $3 \mathrm{ml}$ of blood discarded and $7.5 \mathrm{ml}$ collected for clotting studies and platelet counting.
The cannula was reconnected to the pump and flushed clear of blood with the acid citrate dextrose solution.

In rabbits pretreated with aspirin, adequate drug induced inhibition of platelet function was assessed by testing in vitro platelet aggregation to ascitic fluid collagen before study. ${ }^{8}$

\section{COAGULATION STUDIES ON PATIENTS \\ UNDERGOING PERITONEOVENOUS SHUNTING FOR \\ REFRACTORY ASCITES}

Seven patients undergoing a total of eight peritoneovenous shunt insertions were studied. There were four men and three women ranging in age from 23 to 54 years. Four patients had chronic liver disease (alcoholic cirrhosis in two, chronic active hepatitis in one, and Budd Chiari syndrome in one) and in three patients ascites was secondary to peritoneal seeding from a malignant neoplasm (gastric in one, pancreatic in one, and colonic in the third). The clinical features of the seven patients studied are detailed in Table 1. Ascitic fluid was obtained from each patient by abdominal paracentesis using a 22 gauge needle and assessed for its ability to aggregate human platelets as previously described. ${ }^{8}$ The day before surgery, each patient was started on aspirin suppositories $300 \mathrm{mg}$ once daily and dipyridamole $100 \mathrm{mg}$ six hourly orally. Antiplatelet therapy was continued throughout the study. Adequate inhibition of platelet response to collagen was tested by in vitro platelet aggregation studies before surgery using a commercial collagen preparation in a final concentration of $5 \mu \mathrm{g} / \mathrm{ml}$ (Hormon-Chemie, Munich). Postoperatively, platelet counts and coagulation studies were performed daily for one week. Each patient gave written informed consent and the study was approved by the local Ethics Review Committee.

\section{Results}

\section{ANIMAL EXPERIMENTS}

The partially purified ascitic fluid collagen suspension in a final dilution of 1:20 caused the irreversible aggregation of human and rabbit platelets in vitro. Prior incubation of the collagen preparation with highly purified collagenase form III (Advance Biofactures Corporation, NY) resulted in complete inhibition of aggregation. ${ }^{8}$ All six rabbits infused with collagen suspension developed changes compatible with disseminated intravascular coagulation (Table 2). Thrombocytopenia was the earliest event (recorded in the first postinfusion sample) and persisted throughout the experiment. The prothrombin and activated partial thromboplastin times were significantly prolonged and there 
Table 1 Clinicial characteristics of patients and results of shunt insertion

\begin{tabular}{|c|c|c|c|c|c|c|c|}
\hline Patient & Age & $\operatorname{Sex}$ & Clinical diagnosis & $\begin{array}{l}\text { Albumin } \\
35-40 \mathrm{~g} / l^{*}\end{array}$ & $\begin{array}{l}\text { Bilirubin } \\
<23 \mu \mathrm{mol} / l^{*}\end{array}$ & $\begin{array}{l}\text { Clinical } \\
\text { bleeding } \\
\text { postshunt }\end{array}$ & Outcome \\
\hline 1 & 42 & $\mathbf{M}$ & Metastatic pancreatic carcinoma & 30 & 13 & None & Ascites resolved \\
\hline 2 & 23 & $\mathbf{M}$ & $\begin{array}{l}\text { Chronic active hepatitis, } \\
\text { cholangiocarcinoma }\end{array}$ & 16 & 200 & $\begin{array}{l}\text { Bleeding } \\
\text { oesophageal } \\
\text { varices }\end{array}$ & Died \\
\hline 4 & 54 & $\mathbf{M}$ & Alcoholic cirrhosis & 29 & 8 & None & Ascites resolved \\
\hline 5 & 47 & $\mathbf{F}$ & Metastatic colonic carcinoma & 31 & 8 & None & Ascites resolved \\
\hline 6 & 35 & $\mathbf{M}$ & Metastatic gastric carcinoma & 30 & 13 & None & Ascites resolved \\
\hline 7 (a) & 40 & $\mathbf{F}$ & $\begin{array}{l}\text { Alcoholic cirrhosis, } \\
\text { hepatorenal syndrome }\end{array}$ & 27 & 116 & None & Ascites resolved \\
\hline (b) & 40 & $\mathbf{F}$ & $\begin{array}{l}\text { Alcoholic cirrhosis, } \\
\text { hepatorenal syndrome }\end{array}$ & 33 & 70 & None & Ascites resolved \\
\hline
\end{tabular}

* Normal values.

was a reduction in the levels of fibrinogen, antithrombin III and factors VIII and IX clotting activities. Fibrin monomers were detected in all six rabbits. The changes in the coagulation studies were maximal, 90 minutes postinfusion.

Platelets from the six rabbits pretreated with aspirin failed to respond in vitro to the ascitic fluid collagen suspension diluted 1:20 (Table 2). At higher concentrations (1:5 dilution) some platelet aggregation was observed. In these animals the collagen infusion was associated with little alteration in the platelet counts or coagulation results.

In the two rabbits infused with normal saline, the clotting studies and platelet counts remained unchanged.

Table 2 Coagulation parameters pre-and postascitic fluid collagen infusion in rabbits

\begin{tabular}{|c|c|c|c|c|}
\hline \multirow[b]{2}{*}{ Coagulation test } & \multicolumn{2}{|c|}{ Untreated rabbits } & \multicolumn{2}{|c|}{$\begin{array}{l}\text { Aspirin treated } \\
\text { rabbits }\end{array}$} \\
\hline & Pre & Post & Pre & Post \\
\hline PT (sec) & $8 \cdot 8 \pm 0 \cdot 4$ & $10 \cdot 4 \pm 1 \cdot 5^{*}$ & 9 & 9 \\
\hline PTTK (sec) & $37 \pm 4$ & $53 \pm 10 \ddagger$ & $36 \pm 6$ & $36 \pm 6$ \\
\hline TT (sec) & $20 \pm 10$ & $22 \pm 10$ & 21 & 21 \\
\hline VIIIc (\% activity) & $101 \pm 4$ & $84 \pm 22$ & $103 \pm 4$ & $100 \pm 6$ \\
\hline IXc (\% activity) & $102 \pm 5$ & $45 \pm 5 \ddagger$ & $100 \pm 6$ & $95 \pm 4$ \\
\hline ATIII (\% activity) & $100 \pm 5$ & $70 \pm 6 \ddagger$ & $90 \pm 14$ & $85 \pm 12$ \\
\hline Fibrinogen (mg\%) & $360 \pm 24$ & $160 \pm 46 \ddagger$ & $350 \pm 20$ & $310 \pm 60$ \\
\hline Platelets $\left(\times 10^{9} / 1\right)$ & $311 \pm 108$ & $161 \pm 79+$ & $328 \pm 80$ & $297 \pm 81$ \\
\hline Fibrin monomers & - & ++ & - & + \\
\hline
\end{tabular}

PT = prothrombin time, PTTK = partial thromboplastin time with kaolin. TT $=$ thrombin time.

Results are mean \pm 2 SD of six different studies.

${ }^{*} p<0.05, \neq p<0.01, \neq p<0.001$. Statistical significance compared with preinfusion result calculated using Student's $t$ test.
STUDIES ON PATIENTS WITH REFRACTORY ASCITES UNDERGOING PERITONEOVENOUS SHUNTING

A total of eight shunts in seven patients were studied. Preoperatively the four patients with chronic liver disease had abnormal coagulation profiles with findings consistent with disseminated intravascular coagulation in two. The three patients with malignant ascites had normal preoperative clotting results. Except for mild thrombocytopenia in one patient with liver disease, all other patients had either normal (five patients) or increased (one patient) platelet counts.

In all patients their ascitic fluid was concentrated and was shown to contain platelet aggregating activity. ${ }^{8}$ Preoperatively, their platelets failed to aggregate to collagen in vitro indicating adequate antiplatelet therapy.

Postoperatively mild thrombocytopenia developed in one patient, and persisted in another. In all the others the platelet count remained within the normal range. Fibrin monomers and fibrin degradation products were detected in all patients. After five shunts there were no other major alterations in the clotting profile and in one instance the clotting parameters actually improved. In only two patients (nos 2 and 3) was there evidence of further impairment of haemostasis postshunt. One had preoperative evidence of moderate disseminated intravascular coagulation which increased after surgery. This patient had cholangiocarcinoma and established cirrhosis secondary to chronic active hepatitis. He succumbed to bleeding oesophageal varices and at necropsy his liver was shown to be extensively replaced by tumour tissue. In spite of active disseminated intravascular coagulation, as indicated by further prolongation of clotting times and a decrease in plasma fibrinogen, he had no other 
clinical evidence of impaired haemostasis. The second patient who developed disseminated intravascular coagulation had Budd Chiari syndrome and developed a postoperative septicaemia which probably contributed to the deterioration in her clotting profile. Table 3 details the pre- and postshunt laboratory investigations on the seven patients studied.

Excluding the patient who bled from oesophageal varices, clinical bleeding was not observed in any of the patients and no instance of immediate shunt occlusion was encountered. One patient developed delayed shunt thrombosis two months postsurgery.

\section{Discussion}

Peritoneovenous shunts are most useful in the management of severe ascites refractory to medical therapy. The underlying pathology is commonly advanced liver disease or peritoneal seeding from malignant neoplasms and these patients in general tend to have critically balanced haemostatic mechanisms. In a number of studies, disseminated intravascular coagulation has been the major postoperative complication requiring shunt ligation for control and limiting the use of the procedure to selected patients with normal haemostasis. ${ }^{1-4}$ Heparin has been suggested as potentially useful in the management of disseminated intravascular coagulation in these patients. ${ }^{2}$ The use of this drug in thrombocytopenic patients with liver disease and disturbed haemostasis, however, carries considerable risks and is best avoided.
The pathogenesis of disseminated intravascular coagulation postperitoneovenous shunt has lately been the subject of a number of studies. Proposed mechanisms that have been debated include the presence in ascitic fluid of procoagulant activity; possibly thromboplastin, ${ }^{6}$ endotoxin, ${ }^{13}$ or cellular. ${ }^{5}$ We have recently reported on the presence of a platelet aggregating factor in ascitic fluid obtained from patients with chronic liver disease and malignant ascites ${ }^{8}$ and identified this as being collagen. As collagen has been well documented to activate contact clotting factors and also cause platelet aggregation ${ }^{14}$ it was suggested that it could play a role in the pathogenesis of disseminated intravascular coagulation postperitoneovenous shunt, and that antiplatelet drugs may have a potential role in the prevention of this complication.

The results of this study indicate that collagen with potent platelet aggregating activity can be purified from ascitic fluid. When infused into rabbits it results in moderate to severe disseminated intravascular coagulation with significant thrombocytopenia. Rabbits pretreated with aspirin were significantly protected from the effects of collagen on the platelet count and coagulation times. The beneficial effects of aspirin are most probably related to the antiplatelet activity of the drug, namely the acetylation of platelet cyclooxygenase (an essential enzyme in the prostaglandin pathway) and thus the inhibition of collagen induced platelet activation. ${ }^{15}$ In the absence of activated platelets the rate of contact clotting factor activation and thrombin generation would be expected to be significantly

Table 3 Results of coagulation profile pre-and postshunt insertion

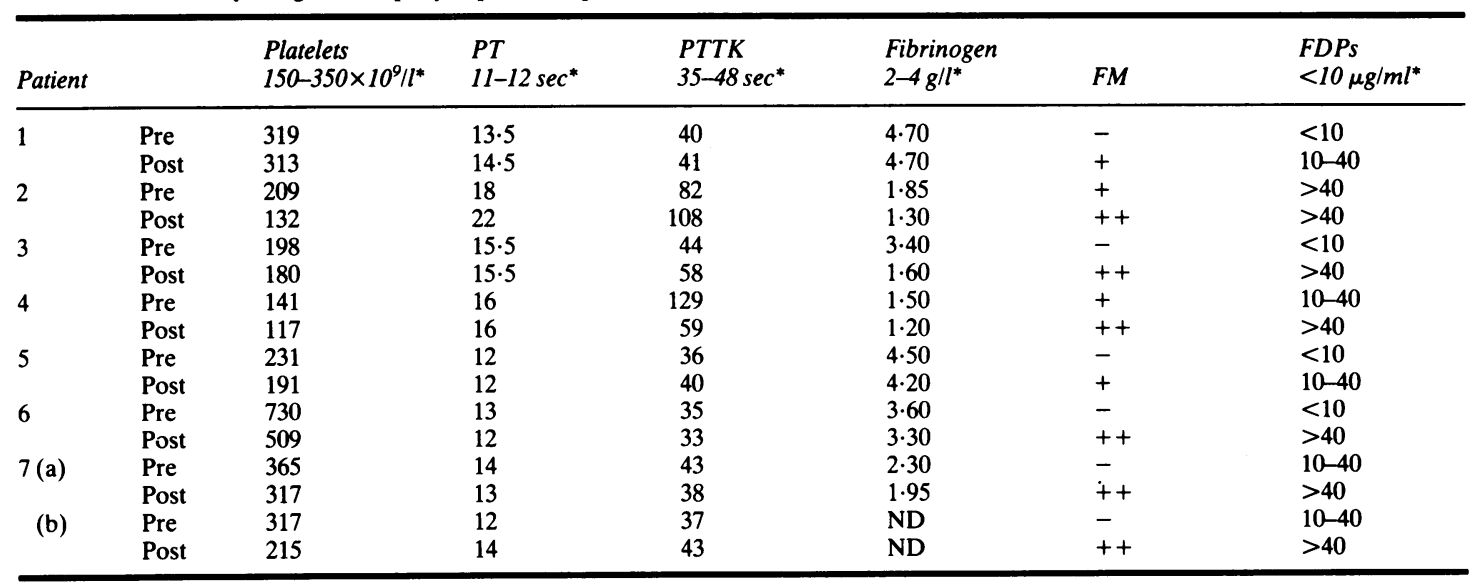

PT = prothrombin time, PTTK = partial thromboplastin time with kaolin, FDPs $=$ fibrin degradation products, ND = not done

$F M=$ fibrin monomers.

* Normal values. 
reduced, ${ }^{16}$ allowing the neutralisation of free thrombin by plasma antithrombins, the hepatic synthesis of consumed clotting factors, and the clearance of collagen and activated clotting factors by the reticuloendothelial system.

Dipyridamole, which inhibits platelet function by increasing platelet cyclic AMP concentrations, is known to act synergistically with aspirin and the potential of this combination to limit disseminated intravascular coagulation complicating peritoneovenous shunting was explored in an uncontrolled study. After shunt surgery fibrin monomers and degradation products were detected in all patients suggesting in vivo activation of the clotting system. Ascitic fluid, however, is rich in both FDPs and fibrin monomers ${ }^{17}$ and their detection in the blood, postshunt, cannot be considered diagnostic of intravascular coagulation in the absence of supporting data. Their presence may purely reflect shunt patency and function. Other evidence of disseminated intravascular coagulation, such as clinical bleeding, thrombocytopenia, prolongation of clotting times, or a decrease in plasma fibrinogen, was not apparent after shunt surgery in six of the eight patients pretreated with aspirin and dipyridamole. Even if the clotting system was activated in these patients it was well compensated with maintenance of prothrombin and partial thromboplastin times and plasma fibrinogen levels. These results contrast with our previous experience in a similar group of patients in whom a patent shunt resulted in a $100 \%$ incidence of disseminated intravascular coagulation associated with significant changes in platelet counts, clotting times and plasma fibrinogen, and a $50 \%$ incidence of clinical bleeding. ${ }^{1}$ Of the two patients in the present series who developed decompensated disseminated intravascular coagulation postperitoneovenous shunt other factors may have contributed to the deterioration of their haemostatic profile. One patient had pre-existing disseminated intravascular coagulation and severe hepatocellular failure and the other developed postoperative septicaemia which may have been involved in the genesis of her consumptive coagulopathy. Neither of these patients developed generalised bleeding although one bled from oesophageal varices.

Early shunt thrombosis has been suggested to reflect the severity of intravascular coagulation postshunting. ${ }^{2}$ It is thus noteworthy that in this current study, early shunt thrombosis was not observed. In our previous experience, where disseminated intravascular coagulation was florid, early shunt occlusion occurred in $40 \%$ of the shunts performed. ${ }^{1}$

In conclusion, we have shown that collagen with potent platelet aggregating activity can be purified from ascitic fluid and when injected into rabbits results in the development of intravascular coagulation. Rabbits pretreated with aspirin were protected from the effects of collagen on platelets and clotting factors. We have also presented preliminary evidence that the use of aspirin and dipyridamole in man may protect against the coagulopathy of peritoneovenous shunts. These observations warrant a double blind controlled study examining the efficacy of antiplatelet therapy in the prevention of this major complication of peritoneovenous shunts.

This study was supported by The Anticancer Council of Victoria; the Life Insurance Medical Research Fund of Australia and New Zealand; and The Alfred Hospital Whole Time Medical Specialists Private Practice Scheme.

\section{References}

1 Gibson PR, Dudley FJ, Jakobovits AW, Salem HH, McInnes IE. Disseminated intravascular coagulation following peritoneovenous (Le Veen) shunt. Aust $N \mathrm{Z}$ J Med 1981; 11: 8-12.

2 Harmon DC, Demirjian Z, Ellman L, Fischer JE. Disseminated intravascular coagulation with the peritoneovenous shunt. Ann Intern Med 1979; 90: $774-6$.

3 Stein SF, Fulenwider JT, Ansley JD et al. Accelerated fibrinogen and platelet destruction after peritoneovenous shunting. Arch Intern Med 1981; 141: 1149-51.

4 Ansley JD, Bethel RA, Bowen PA, Warren WD. Effect of peritoneovenous shunting with the Le Veen valve on ascites, renal function, and coagulation in six patients with intractable ascites. Surgery 1978; 83: 181-7.

5 Lerner RG, Nelson JC, Corines P, del Guercio LRM. Intravascular coagulation complicating peritoneal atrial shunts. (Abstract.) Blood 1977; 50 suppl I: 274.

6 Johnston MFM, Vargo J, Joist JH. Isolation and characterization of procoagulant substances from human ascites. (Abstract.) Thromb Haemostas 1981; 41 suppl 1: 353 .

7 Giles AR, Sauder D, Seaton TL, Johnston M, Hirsch J. Changes in the coagulation status of patients undergoing autotransfusion of concentrated ascitic fluid as treatment of refractory ascites. (Abstract.) Blood 1977; 50 suppl I: 267.

8 Salem HH, Koutts J, Handley C, Van Der Weyden MB, Dudley FJ, Firkin BG. The aggregation of human platelets by ascitic fluid: a possible mechanism for disseminated intravascular coagulation complicating Le 
Veen shunts. Am J Hematol 1981; 11: 153-7.

9 Trelstad RL, Catanese VM, Rubin DF. Collagen fractionation: separation of native types I, II and III by differential precipitation. Anal Biochem 1976; 71: 114-8.

10 Lowry OH, Rosenrough NJ, Farr AL, Randall RS. Protein measurement with the folin phenol reagent. $J$ Biol Chem 1951; 193: 265-75.

11 Biggs R. Human blood coagulation, haemostasis and thrombosis, 2nd ed. Oxford: Blackwell, 1976.

12 Odegard OR. Evaluation of an amidolytic heparin cofactor assay method. Thromb Res 1975; 7: 351-60.

13 Murr H, Schussler P, Rindfleisch GE, Eisenburg J, Hiller E. Endotoxins and procoagulant activities of ascitic fluid. (Abstract.) Thromb Haemostas 1981; 41: 232.

14 Walsh PN. The effects of collagen and kaolin on the intrinsic coagulant activity of platelets. $\mathrm{Br} \mathrm{J}$ Haematol 1972; 22: 393-405.

15 Jobin F. Acetylsalicylic acid, hemostasis and human thromboembolism. Semin Thromb Hemostas 1978; 4: 199-240.

16 Majerus PW, Miletich JP. Relationship between platelets and coagulation factors in hemostasis. Ann Rev Med 1978; 29: 41-9.

17 Hoefs J, Barnes T, Halle P. Intraperitoneal coagulation in chronic liver disease ascites. Dig Dis Sci 1981; 26: 518-22. 